\title{
Utilidad del ultrasonido a la cabecera del paciente con neumotórax en infección por SARS-CoV-2. Reporte de caso
}

\author{
Usefulness of bedside ultrasound in patients with \\ pneumothorax in SARS-CoV-2 infection. Case report
}

Ancizar Joaquín de la Peña Silva1, Yerlin Andrés Colina Vargas², Jorge Eduardo Bejarano Botero²

\begin{abstract}
It has been described that patients with coronavirus disease 2019 (COVID-19) may present pneumothorax, either spontaneously, primarily due to constant cough, secondary to pneumonia caused by said disease, or iatrogenic due to mechanical ventilation that require high pressures, or traumatic due to urgent approaches, in a difficult airway such as that of these patients. The use of bedside ultrasonography has been gaining great relevance to complement clinical diagnosis, which has become a useful and reliable tool for personnel treating critically ill patients.
\end{abstract}

\section{RESUMEN}

Se ha descrito que los pacientes con la enfermedad por coronavirus 2019 (COVID-19), pueden presentar neumotórax, ya sea de forma espontánea, primaria por la tos constante, secundaria a la neumonía causada por dicha enfermedad, o ya sea iatrogénica por la ventilación mecánica que requieren con altas presiones, o traumática por los abordajes urgentes, en una vía aérea difícil como la de estos pacientes. El uso de la ultrasonografía a la cabecera del paciente ha venido cobrando gran relevancia para complementar el diagnóstico clínico, la cual se ha convertido en una herramienta útil y fiable para el personal que atiende pacientes críticamente enfermos.

\section{Key words:}

Ultrasonography, pneumothorax, obesity, coronavirus infections

\section{Palabras clave:}

Ultrasonografía, neumotórax, obesidad, infecciones por coronavirus

Médico Anestesiólogo

Médico Intensivista. Clínica Universitaria Bolivariana. Medellín, Colombia.

Fecha de recepción: 28 de enero de 2021

Fecha de aceptación: 20 de febrero de 2021

\section{ORCID}

0000-0002-0581-288X

\section{Correspondencia:}

Yerlin Andrés Colina Vargas

colinavargas1910@gmail.com 
Ultrasonido en neumotórax - A. J. de la Peña S. et al.

\section{Introducción}

a enfermedad por coronavirus 2019 (COVID-19) es causada por el coronavirus 2 del virus del síndrome respiratorio agudo severo (SARS-CoV-2), que se ha convertido en pandemia desde 2019, surgido en Wuhan, provincia de Hubei, China[1]. Los principales hallazgos son: fiebre, tos y dificultad para respirar; entre otros síntomas posibles están: dolor abdominal, mialgia, diarrea, dolor de garganta, fatiga y anosmia[2]. El 81\% desarrolla síntomas leves, $14 \%$ severos y $5 \%$ progresa a condición crítica por falla respiratoria, choque séptico o falla orgánica múltiple[3], requiriendo la gran mayoría ventilación mecánica invasiva prolongada, soporte vasopresor, posicionamiento en prono y relajación neuromuscular[4].

El neumotórax es una entidad clínica definida como la presencia de aire entre la pleura visceral y parietal, que puede afectar la oxigenación y ventilación. Se clasifica en: espontáneo (primario o secundario), traumático o iatrogénico, siendo más común el espontáneo, el cual, cuando se trata del secundario se produce debido a una enfermedad pulmonar preexistente, por lo que la tensión severa durante la tos persistente o ventilación mecánica con presión positiva en la neumonía COVID-19 puede ser el factor causante del neumotórax[3]. Los beneficios de las técnicas de diagnóstico rápido, como la ecografía a la cabecera del paciente, son importantes en el manejo del paciente crítico y se reconocen cada vez más en el tratamiento[4],[5].

\section{Presentacion del caso}

Femenina de 67 años, con IMC de $43,5 \mathrm{~kg} / \mathrm{m}^{2}$ y diagnóstico de neumonía por SARS-CoV-2, con tubo endotraqueal (TET) 8,5 mm, en protocolo de pronación y relajación neuromuscular, ventilación mandatoria continua controlada por presión (VMC-P), con PC: $25 \mathrm{cmH}_{2} \mathrm{O}$ FR: 23/min $\mathrm{FiO}_{2}$ : $100 \%$ PEEP: $12 \mathrm{cmH}_{2} \mathrm{O}$ con monitoria de presión pico: $37 \mathrm{cmH}_{2} \mathrm{O}$ VC: $258 \mathrm{ml}$ Vmin: 6,2 l/min presión meseta: $31 \mathrm{cmH}_{2} \mathrm{O}$ presión de conduccion:19 $\mathrm{cmH}_{2} \mathrm{O}$ resistencia inspiratoria:13 $\mathrm{cmH}_{2} \mathrm{O} / / / \mathrm{s}$ compliance estática: $13 \mathrm{ml} / \mathrm{cmH}_{2} \mathrm{O}$.

Solicitan el servicio de anestesiología para cambio de TET por neumotaponador roto y fugas mayores al 50\% del VC. En historia clínica se reportó primera intubación difícil con videolaringoscopia. Se plantea cambio de TET con FROVA $14 \mathrm{fr}$, se desliza hasta 35 cm del adaptador del TET y bajo visión con laringoscopia directa se retira el TET. Se desliza el nuevo TET 8,5 $\mathrm{mm}$ fácilmente, se fija a $24 \mathrm{~cm}$ de la comisura oral, se retira el FROVA y se conecta al ventilador mecánico.

Aumentaron las presiones en vía aérea, bajó el VC y se deterioró la $\mathrm{SaO} 2$, por lo que se pasa a ventilación manual con BVM, percibiendo ventilación difícil, con mucha resistencia. En la auscultación murmullo vesicular disminuido globalmente. Se inicia manejo por probable broncoespasmo con salbutamol, y sulfato de magnesio; sin mejoría, con posterior deterioro hemodinámico. En la inspección, aparente asimetría derecha, pero por el tamaño de las mamas, fue difícil asegurarlo. Se realiza ultrasonido pulmonar en ambos ápices y se evidencia ausencia del deslizamiento pulmonar derecho en el modo B y signo del código de barras en el modo M (Figura 1), confirmando neumotórax, realizamos inmediatamente descompresión torácica con catéter 14G, puncionando en segundo espacio intercostal, línea medio clavicular derecha, se percibe salida de aire y mejoría clínica recuperando la $\mathrm{SaO} 2$ a $90 \%$ y estabilidad hemodinámica, se realiza eco inmediatamente con resolución del neumotórax dado por hallazgos de deslizamiento pleural y se evidencia derrame pleural, se solicita asistencia a cirugía general para implantación de sonda de toracostomía.

\section{Discusión}

En el informe de Yao et al., que caracterizó 202 pacientes COVID-19 en Wuhan, reportaron una incidencia de 5,9\% neumotórax, considerando que la estructura pulmonar alterada por la inflamación en fase tardía de la enfermedad y las maniobras ventilatorias que generan altas presiones en la vía aérea predisponen al desarrollo del evento. Surgiendo, posteriormente, más reportes de neumotórax asociados al COVID-19[6]-[8].

En cuanto a la vía aérea del paciente con COVID-19, se considera difícil por la gravedad de la hipoxemia, las dificultades derivadas del uso de equipo de protección personal y el entorno laboral desafiante. Adicionalmente, los pacientes obesos se consideran de difícil manejo de vía aérea, sumado a su factor de riesgo para desarrollar COVID-19 y de mayor gravedad; cumplen criterios de intubación y ventilación difícil, requiriendo medidas adicionales. Abou-Arab y col. describieron en dos casos, que el uso de intercambiador probablemente pudo haber generado un trauma traqueal, empeorando la condición respiratoria y requiriendo ECMO urgente. La ausencia de visibilidad de la glotis en ambos pacientes pudo aumentar el riesgo de traumatismo traqueal al forzar la intubación con el intercambiador. Es recomendable siempre la videolaringoscopia no solo para la protec- 


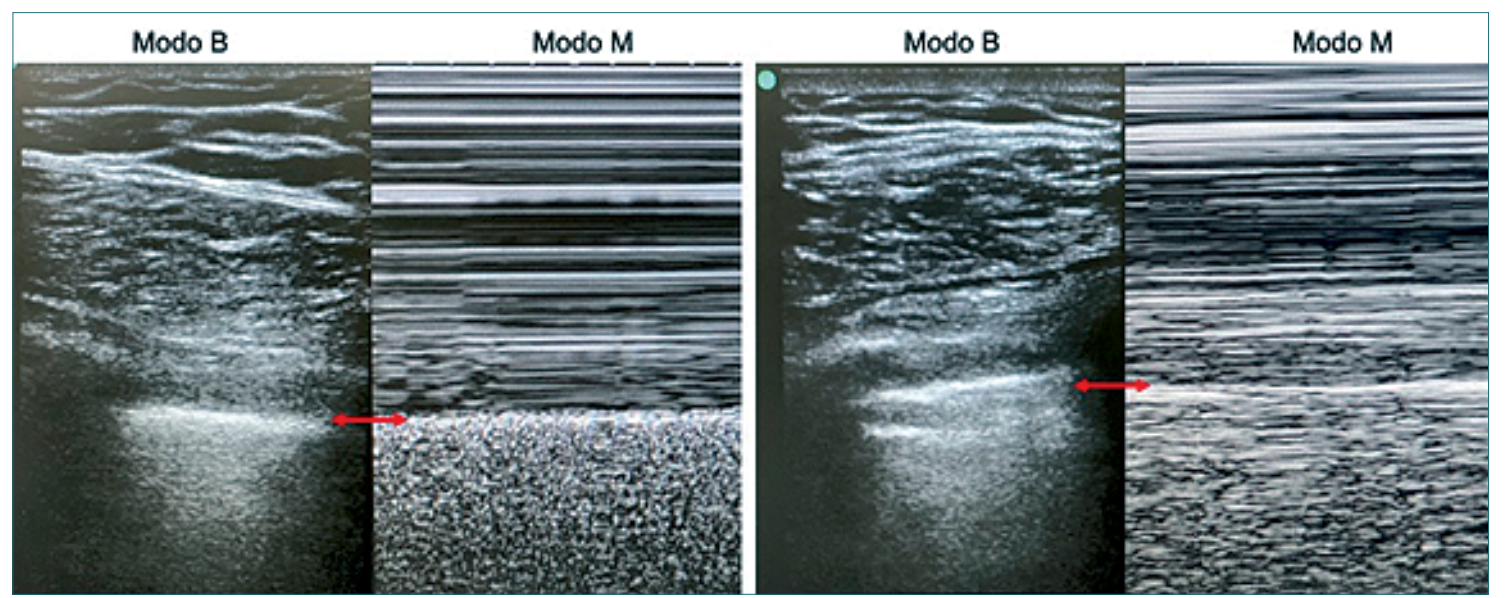

Figura 1. Imagen ecográfica normal y con hallazgo de neumotórax. A. Hallazgo normal. Signo de la arena y el mar; B. Hallazgo de neumotórax. Signo del código de barras.

ción del personal sanitario, sino también para permitir una intubación exitosa en el primer intento, evitando posible traumatismo traqueal y empeoramiento de la insuficiencia respiratoria[9],[10].

En la mayoría de pacientes con COVID-19, la intubación traqueal se puede realizar de forma semielectiva, dando tiempo para evaluar al paciente, preparar equipo y discutir la estrategia de manejo de la vía aérea; incluyendo el posicionamiento y uso primario de videolaringoscopia dada su eficacia y menor riesgo de contaminación. La preoxigenación es importante en pacientes obesos hipoxémicos, la optimización del suministro de oxígeno antes de la intubación permite un manejo más controlado y minimiza la probabilidad de eventos adversos[10].

Al presentarse un trauma en las vías aéreas, se recomienda evaluación multidisciplinaria; donde un enfoque conservador sería preferible, especialmente en pacientes críticos con COVID-19 cuando esté indicado (desgarros traqueales menores por encima del nivel del manguito del tubo traqueal), lesión de la membrana cricotiroidea inducida por el tubo, neumotórax y neumomediastino. Es importante no forzar el avance ya sea cuando se enfrentan vistas laríngeas limitadas o cuando ha pasado las cuerdas vocales. Cualquier avance más allá de la carina acarrea alto riesgo de traumatismo de vías respiratorias; por tanto, no debe insertarse más de $8 \mathrm{~cm}$ después de las cuerdas. Estas precauciones pueden ser incluso más relevantes en pacientes COVID-19, cuyas vías respiratorias inflamadas pueden ser más propensas a traumatismos[10]-[12]. Dado lo anterior, es importante considerar el neumotórax a tensión como una causa de deterioro agudo en pacientes con infección por SARS-CoV-2. La importancia de diagnosticar correctamente la patología pulmonar secundaria, como consecuencia del COVID-19, radica en el abordaje terapéutico diferente requerido para los dos escenarios, con consecuencias potencialmente mortales si se elige el manejo incorrecto[11],[12].

La ecografía pulmonar es una técnica sencilla de cabecera con numerosas aplicaciones. Puede ayudar a los médicos en el diagnóstico de los principales trastornos respiratorios que afectan al enfermo crítico, sugiriendo así el abordaje terapéutico en urgencias y UCl. Se puede utilizar para evaluar y controlar la aireación pulmonar en paciente con insuficiencia respiratoria aguda y puede ser una herramienta útil para guiar la ventilación mecánica y varios procedimientos, como maniobras de reclutamiento, pronación, fibrobroncoscopia y drenaje pleural[4],[5]. La ecografía a la cabecera del paciente se utiliza cada vez más, para integrar la evaluación clínica de los enfermos críticos; en particular, la ecografía pulmonar se ha desarrollado mucho en la última década. La semiótica pulmonar se compone de artefactos (derivados de la interfaz aire/ tejido) e imágenes reales (es decir, derrames y consolidaciones) que proporcionan información para identificar los principales trastornos respiratorios agudos. Los signos de ultrasonido pulmonar, solos o combinados con otras técnicas de ultrasonido, son útiles en el enfoque diagnóstico de pacientes con insuficiencia respiratoria aguda, shock circulatorio o paro cardíaco. Se puede realizar una semicuantificación de la aireación pulmonar al lado de la cama y usarse en pacientes con ventilación mecánica para guiar el ajuste de la presión espiratoria positiva, evaluar la eficacia de los tratamientos, monitorear la evolución del trastorno 
respiratorio y ayudar en el proceso de destete. Es útil para la detección precoz y el tratamiento de complicaciones respiratorias bajo ventilación mecánica, como neumotórax, neumonía asociada al ventilador, atelectasia y derrames pleurales; es una herramienta útil de diagnóstico y seguimiento que podría, en un futuro próximo, formar parte del conocimiento básico de los médicos que atienden al paciente crítico[4],[5].

Los beneficios de las técnicas de diagnóstico rápido, como la ecografía a la cabecera del paciente, son muy importantes en el manejo del paciente crítico y se reconocen cada vez más en el tratamiento de COVID-19. Los pacientes que presentan insuficiencia respiratoria, con frecuencia se presumen COVID-19 positivos hasta que se demuestre lo contrario, sin embargo, el examen minucioso y la perspicacia clínica corren el riesgo de ser reemplazados por estrategias de tratamiento basadas en protocolos, los cuales, suelen recomendar que, todos los pacientes con sospecha de COVID-19 y $\mathrm{SpO}_{2}<94 \%$ con alto flujo de oxígeno $\left(60 \%\right.$ de $\left.\mathrm{FiO}_{2}\right)$ deben comenzar de inmediato con CPAP dentro de los 10 min de la presentación. Si se siguiesen dichas recomendaciones, podría empeorar el escenario clínico de dichos pacientes que cursen con neumotórax. Por tanto, es importante evitar la adopción de un enfoque puramente algorítmico para los pacientes críticamente enfermos[11],[12].

Finalmente, en el presente reporte, mostramos cómo el neumotórax tiene un origen multifactorial en el escenario del paciente COVID-19, pudiendo desencadenarse de forma espontánea por la misma clínica (tos persistente, por ejemplo), de forma secundaria a la neumonía generada por la enfermedad, iatrogénica por la ventilación mecánica o traumática por el uso de intercambiadores de tubos. No es común que el talento humano en salud reporte los propios eventos adversos, posiblemente por miedo a las consecuencias sobre la reputación, o miedo a las consecuencias legales[10]-[12]. Por lo que es habitual el sesgo de publicación favoreciendo los informes con resultados positivos[13]. En cualquiera de los tres escenarios potenciales del neumotórax de esta paciente, sin duda, el médico tiene inicialmente el reto de establecer un diagnóstico rápido, de ahí que la utilización del ultrasonido dentro del cubículo facilita este objetivo, por otro lado, debe implementar una estrategia de solución salvadora como la descompresión torácica con aguja hasta que pueda establecerse una medida definitiva como la sonda de toracostomía[13].

\section{Conclusiones}

No es recomendable que la práctica médica se rija exclusivamente por algoritmos, cada paciente es diferente, y por ende no debemos perder nuestra pericia clínica para el abordaje. Dadas las condiciones de inflamación del tejido pulmonar de pacientes COVID-19, sus comorbilidades y los escenarios desafiantes de la ventilación mecánica se debe tener alta sospecha del neumotórax como complicación durante el manejo y considerar el ultrasonido como excelente herramienta, usualmente disponible, para el diagnóstico a la cabecera del paciente de múltiples condiciones clínicas, en este caso, un neumotórax, que de no haberse diagnosticado y manejado oportunamente, pudo ser fatal.

\section{Referencias}

1. Hui DS, I Azhar E, Madani TA, Ntoumi F, Kock R, Dar O, et al. The continuing 2019-nCoV epidemic threat of novel coronaviruses to global health - The latest 2019 novel coronavirus outbreak in Wuhan, China. Int J Infect Dis. 2020 Feb;91:264-6. https://doi. org/10.1016/j.ijid.2020.01.009 PMID:31953166

2. Ucpinar BA, Sahin C, Yanc U. Spontaneous pneumothorax and subcutaneous emphysema in COVID-19 patient: case report.
J Infect Public Health. 2020

Jun;13(6):887-9. https://doi. org/10.1016/j.jiph.2020.05.012 PMID:32475804

3. Yao $W$, Wang $T$, Jiang $B$, Gao F, Wang L, Zheng $H$, et al.; collaborators. Emergency tracheal intubation in 202 patients with COVID-19 in Wuhan, China: lessons learnt and international expert recommendations. Br J Anaesth. 2020 Jul;125(1):e28-37. https://doi. org/10.1016/j.bja.2020.03.026 PMID:32312571

4. Dahmarde H, Parooie F,
Salarzaei M. Accuracy of Ultrasound in Diagnosis of Pneumothorax: A Comparison between Neonates and Adults-A Systematic Review and MetaAnalysis. Can Respir J. 2019 Dec;2019:5271982. https://doi. org/10.1155/2019/5271982 PMID:31933707

5. Bhatraju PK, Ghassemieh BJ, Nichols M, Kim R, Jerome KR, Nalla AK, et al. Covid-19 in critically ill patients in the Seattle region- case series. $\mathrm{N}$ Engl J Med. 2020 May;382(21):201222. https://doi.org/10.1056/NEJ- 
Moa2004500 PMID:32227758

6. Yao W, Wang $T$, Jiang $B$, Gao F, Wang $L$, Zheng $H$, et al.; collaborators. Emergency tracheal intubation in 202 patients with COVID-19 in Wuhan, China: lessons learnt and international expert recommendations. Br J Anaesth. 2020 Jul;125(1):e28-37. https://doi. org/10.1016/j.bja.2020.03.026 PMID:32312571

7. Shaghaghi S, Daskareh $M_{\text {, }}$ Irannejad M, Shaghaghi M, Kamel IR. Target-shaped combined halo and reversedhalo sign, an atypical chest CT finding in COVID-19. Clin Imaging. 2021 Jan;69:72-4. https://doi.org/10.1016/j. clinimag.2020.06.038 PMID:32682246

8. Rohailla S, Ahmed N, Gough K. SARS-COV-2 infection associated with spontaneous pneumothorax. CMAJ. 2020 May;192(19):E510. https:// doi.org/10.1503/cmaj.200609 PMID:32317275

9. Abou-Arab O, Huette P, Berna P, Mahjoub Y. Tracheal trauma after difficult airway management in morbidly obese patients with COVID-19. Br J Anaesth. 2020 Jul;125(1):e168-70. https://doi. org/10.1016/j.bja.2020.04.004 PMID:32334809

10. Sorbello M, Hodzovic I, Cusumano G, Frova G. Tracheal introducers and airway trauma COVID-19. Comment on $\mathrm{Br}$ J Anaesth 2020; 125: e168e170. Br J Anaesth. 2020 Sep;125(3):e305-7. https://doi. org/10.1016/j.bja.2020.05.013 PMID:32507333

11. Flower $L, C$ arter JL, Rosales López J, Henry AM. Tension pneumothorax in a patient with COVID-19. BMJ Case Rep. 2020 May;13(5):e235861. https://doi. org/10.1136/bcr-2020-235861 PMID:32423911

12. Wu Z, McGoogan JM. Characteristics of and Important Lessons From the Coronavirus Disease 2019 (COVID-19) Outbreak in China: Summary of a Report of 72314 Cases From the Chinese Center for Disease Control and Prevention. JAMA. 2020 Apr;323(13):1239-42. https:// doi.org/10.1001/jama.2020.2648 PMID:32091533

13. Heidegger T. Airway management lessons from case reports of negative outcomes. Comment on $\mathrm{Br} J$ Anaesth; 125: e168-70. Br J Anaesth. 2020 Sep;125(3):e307-9. https://doi. org/10.1016/j.bja.2020.06.042 PMID:32680606 